\title{
Virtual Coiling of Intracranial Aneurysms Based on Dynamic Path Planning
}

\author{
Hernán G. Morales $^{1}$, Ignacio Larrabide ${ }^{1}$, Minsuok Kim ${ }^{1}$, \\ Maria-Cruz Villa-Uriol ${ }^{1}$, Juan M. Macho ${ }^{2}$, Jordi Blasco ${ }^{2}$, \\ Luis San Roman ${ }^{2}$, and Alejandro F. Frangi ${ }^{1}$
}

1 Center for Computational Imaging \& Simulation Technologies in Biomedicine (CISTIB) - Universitat Pompeu Fabra and CIBER-BBN, Barcelona, Spain

${ }^{2}$ Department of Radiology, Hospital Clinic, Barcelona, Spain

\begin{abstract}
Coiling is possibly the most widespread endovascular treatment for intracranial aneurysms. It consists in the placement of metal wires inside the aneurysm to promote blood coagulation. This work presents a virtual coiling technique for pre-interventional planning and post-operative assessment of coil embolization procedure of aneurysms. The technique uses a dynamic path planning algorithm to mimic coil insertion inside a 3D aneurysm model, which allows to obtain a plausible distribution of coils within a patient-specific anatomy. The technique was tested on two idealized geometries: an sphere and a hexahedron. Subsequently, the proposed technique was applied in 10 realistic aneurysm geometries to show its reliability in anatomical models. The results of the technique was compared to digital substraction angiography images of two aneurysms.
\end{abstract}

\section{Introduction}

Endovascular therapies of intracranial aneurysms are an alternative to the traditional surgical clipping. Among them, coiling is the most popular and common option. This treatment consists in the insertion of biocompatible metal wires inside the aneurysm through a catheter. During treatment, angiographic images are produced to guide the catheter and coils while they navigate inside the vasculature. The amount of inserted coils depends on several factors, including the aneurysm morphology, the selected coils and the operator skills, among others. However, clinicians usually try to insert as many coils as possible to achieve high packing densities (defined as the ratio between the inserted coil and aneurysm volume) [1. The goal is to induce through the coils a mechanical resistance against the pulsatile blood flow to promote the hemodynamic conditions that triggers blood coagulation [2].

In recent years, computational methods have appeared as an interesting source of information for clinicians to support them during diagnosis, planning or evaluation of aneurysm treatments. A virtual stenting technique [3] and an interactive simulator for virtual coiling [4] are examples of these methods. In this work, we

G. Fichtinger, A. Martel, and T. Peters (Eds.): MICCAI 2011, Part I, LNCS 6891, pp. 355-362, 2011.

(C) Springer-Verlag Berlin Heidelberg 2011 
present a virtual coiling technique for virtually treating image-based patientspecific cerebral aneurysm models. The proposed method relies on a dynamic path planning algorithm to insert computational models of the coils.

\section{Virtual Coiling Technique}

The proposed virtual coiling technique uses a dynamic path planning algorithm to mimic coil insertion. The description of this algorithm is presented in the following section.

\subsection{The Dynamic Path Planning Algorithm}

A virtual coil model, from here on called a coil, is defined as a set of ordered points (see equation 1). Here, the index $j$ denotes the coil currently under insertion, while the index $i$ corresponds to the points of that coil.

$$
\mathbb{C}_{j} \equiv\left\{\mathbf{p}_{j}^{i} \in \mathbb{R}^{3}: i=0 \ldots n_{j}\right\},
$$

where $n_{j}$ is the number of segments in the coil $j$. This number is defined as the floor function of the ratio between the coil length $\left(L_{j}\right)$ and the coil radius $\left(r_{j}\right)$. For each coil to be inserted both, $L_{j}$ and $r_{j}$ are provided as input to our algorithm.

To add a new point $\mathbf{p}_{j}^{i+1}$ to the coil $\mathbb{C}_{j}$, a set of candidate locations is created as $\mathbb{H}_{j, i} \equiv\left\{\mathbf{h}_{j}^{i} \in \mathbb{R}^{3}:\left\|\mathbf{p}_{j}^{i}-\mathbf{h}_{j}^{i}\right\|=r_{j}\right\}$. The number of candidate locations $\left(\left|\mathbb{H}_{j, i}\right|\right)$ is a parameter of the algorithm, but in general we have set it to over 150 elements to ensure a proper spatial discretization. The $\mathbf{h} \in \mathbb{H}_{j, i}$ with the lowest potential field $\phi(h)$ is selected and included in $\mathbb{C}_{j}$ as $\mathbf{p}_{j}^{i+1}$. This potential field $\phi(h)$ is based on three rules, namely: coiling domain, coil flexibility and coil pull-back, and it is mathematically defined as follows:

$$
\phi(h)=\phi_{D}(h)+\phi_{F}(h)+\phi_{P B}(h) .
$$

Coiling Domain: It represents the region where the coil is allowed to move and it is defined as the sum of two potential fields.

$$
\phi_{D}(h)=\phi_{A}(h)+\phi_{C}(h) .
$$

the first term, $\phi_{A}(h): \mathbb{R}^{3} \rightarrow\{0,1\}$, is defined by the aneurysm model to be filled with coils. If the candidate location $\mathbf{h}$ is inside the model, $\phi_{A}(h)$ is set to zero, otherwise to one (see Fig. 11A). The second term, $\phi_{C}(h): \mathbb{R}^{3} \rightarrow\left\{\left[0, \frac{1}{2}\right), 1\right\}$, avoids crossings of the coils. Also, it accounts for the distance between coils such that newly inserted coils avoid highly dense coiled areas. The field $\phi_{C}(h)$ is defined as follows:

$$
\phi_{C}(h)= \begin{cases}1, & \text { if } \exists \mathbf{m} \text { satisfying }\|\mathbf{h}-\mathbf{m}\| \leq 2 \cdot r_{j}, \\ \frac{r_{j}}{\left|\mathbb{M}_{j, i}\right|} \cdot \sum_{m} \frac{1}{\|\mathbf{h}-\mathbf{m}\|}, & \text { otherwise } .\end{cases}
$$


where $\mathbf{h} \in \mathbb{H}_{j, i}$ and $\mathbf{m} \in \mathbb{M}_{j, i}$. The set $\mathbb{M}_{j, i}$ (see equation [5) is a subset of all points of the inserted coils within a distance of $\alpha \cdot r_{j}$ from the last inserted point of $\mathbb{C}_{j}$, called the coil tip.

$$
\mathbb{M}_{j, i} \equiv\left\{\mathbf{m} \in \bigcup_{q} \mathbb{C}_{q}:\left\|\mathbf{p}_{j}^{i}-\mathbf{m}\right\| \leq \alpha \cdot r_{j}, q=1 \ldots j\right\},
$$

The Euclidean distance, $\alpha \cdot r_{j}$, with $\alpha=4$ was used to limit the size of $\mathbb{M}_{j, i}$, and thereby, to reduce the computational cost of the algorithm. This value of $\alpha$ was set to take into account at least the closest coil points that could potentially produce coil crossings. Figure 1B shows a schematic representation of how $\phi_{C}(h)$ is evaluated.

Coil Flexibility: It takes into account the deformation of the coil while it is inserted, by comparing the angle $\theta$ of the evaluated candidate location with respect to the previous direction $\left(\mathbf{p}_{j}^{i}-\mathbf{p}_{j}^{i-1}\right)$ as it is visualized in figure $1 \mathrm{C}$. The potential field $\phi_{F}(h): \mathbb{R}^{3} \rightarrow\left[0, \frac{1}{2}\right]$ is defined as:

$$
\phi_{F}(h)=\theta /(2 \cdot \pi),
$$

where $\theta=\arccos \left[\left(\mathbf{h}-\mathbf{p}_{j}^{i}\right) \cdot\left(\mathbf{p}_{j}^{i}-\mathbf{p}_{j}^{i-1}\right) / r_{j}^{2}\right]$.

Coil Pull-Back: It is meant to solve the situation when $\phi(h) \geq 1 \forall \mathbf{h} \in \mathbb{H}_{j, i}$ (becoming a dead-end). In such cases the algorithm pulls back and relocates the coil tip to the position $\mathbf{p}_{j}^{i-(k+1)}$, being $k$ the number of consecutive dead-ends. Then, a potential field, $\phi_{P B}=1$ is assigned to the location $\mathbf{p}_{j}^{i+1}$, and thereby, the dead-end is avoided. An schematic example of the coil pull back is presented in figure $1 \mathrm{D}$.
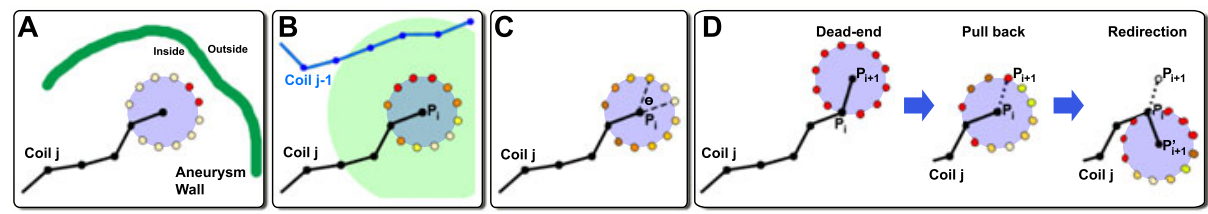

Fig. 1. Two-dimensional schematic description of virtual coiling technique. (A) Rejected candidate locations (red dots) due to $\phi_{A}=1$, since their distance to the aneurysm wall is less than one coil diameter. (B) Evaluation of $\phi_{C}$. The set $\mathbb{M}_{j, i}$ contains the coil points inside the green circle. (C) Evaluation of $\phi_{F}$. (D) Dead-end condition, pull back and redirection of the last coil.

Algorithm Initialization: To initialize $\mathbb{C}_{j}$, a set of initial candidate locations, $\mathbb{S}_{j}$, is defined around the geometrical center of the aneurysm model within a distance of $\beta \cdot r_{j}$, with $\beta=4$. The value of $\beta$ was set to guarantee a sufficient distance between any two initial coil points. Afterwards, the element $\mathbf{s}$ of $\mathbb{S}_{j}$ with the lowest $\phi_{C}(s)+\phi_{F}(s)$ is selected as the first coil point $\mathbf{p}_{j}^{0}$. A user-defined initial direction is set (for instance $\hat{x}$ ), to calculate $\phi_{F}(s)$ during the evaluation of the first two coil points $\mathbf{p}_{j}^{0}$ and $\mathbf{p}_{j}^{1}$. 
The proposed approach is summarized in Algorithm 1.



Algorithm 1. Pseudocode of algorithm for virtual coiling

\section{Test on Idealized Geometries}

We tested how our method behaves inside idealized geometries. For this two geometries were created (see figure 2): a $2 \mathrm{~mm}$-radius sphere, and a $4 \mathrm{x} 4 \mathrm{~mm}^{2}$-base $1.5 \mathrm{~mm}$-height hexahedron with a $0.3 \mathrm{~mm}$-diameter $0.5 \mathrm{~mm}$-height tube above it. Both geometries, sphere and hexahedron, were used to show that the method adapts the shape of the coils to the morphology of the containing geometry, although the hexahedron does not represent a anatomical structure. The small tube on top of the hexahedron was added to force a dead-end and to illustrate how the coil pull-back mechanics operates. This dead-end will appear since the first coil will go straight to the tube end and no chance to bend the coil will be available since the tube diameter would be slightly higher than the coil one.

Five experiments were performed, four of them using the sphere and the last one using the hexahedron. Table 1 summarizes each experiment. The test I and II are meant to see the differences in the coil configuration by changing the coil lengths, maintaining the total coil length $(19 \mathrm{~cm})$. The objective of tests III and IV is to see the influence of the number of candidate locations and to compare the required computational times against the previous experiments. 
Table 1. Experimental set-up on idealized geometries

\begin{tabular}{|c|c|c|c|c|c|c|}
\hline Test & $\begin{array}{c}\text { Idealized } \\
\text { Model }\end{array}$ & $\begin{array}{c}\text { Number of } \\
\text { coils }\end{array}$ & $\begin{array}{c}\text { Coil } \\
\text { diameter [mm] }\end{array}$ & $\begin{array}{c}\text { Coil } \\
\text { lengths [cm] }\end{array}$ & $\begin{array}{c}\text { Number of } \\
\text { candidate locations }\end{array}$ & $\begin{array}{c}\text { Initial } \\
\text { direction }\end{array}$ \\
\hline I & Sphere & 3 & 0.25 & $2-2-15$ & 350 & $\hat{z}$ \\
II & Sphere & 3 & 0.25 & $5-5-9$ & 350 & $\hat{z}$ \\
III & Sphere & 3 & 0.25 & $2-2-15$ & 150 & $\hat{z}$ \\
IV & Sphere & 3 & 0.25 & $5-5-9$ & 150 & $\hat{z}$ \\
V & Hexahedron & 3 & 0.25 & $2-2-20$ & 350 & $\hat{z}$ \\
\hline
\end{tabular}

Note that, although clinicians first insert the longest coils, here the shortest ones were placed before to show how the coils are initiated and to have a better idea of the algorithm functioning when the models are almost empty.

\subsection{Results on Idealized Models}

Figure 2A, B and $\mathrm{C}$ present the result of Test $\mathrm{I}$ with one, two and the three coils, respectively. The obtained packing density with these coils was $27.3 \%$. The result of Test II is visualized in figure $2 \mathrm{D}$, where a different coil configuration was obtained compared to Test I, although the same high packing density of $27.3 \%$ was achieved. Figure $2 \mathrm{E}$ and $\mathrm{F}$ showed the results of Test II and IV, respectively. As it is visualized, the reduction of candidate locations produces different coil configurations, and the coil roughness increased (sharper angles). The same packing density of $27.3 \%$ was obtained with these two experiments.

Test I and II required 230s each to be computed in a Intel Core(TM)2 Quad CPU Q6600 @2.4GHz with 8 GB of RAM. This time was reduced to 93 seconds in Test III and IV, due to the reduction of candidate locations.

The results of Test $\mathrm{V}$ are presented in figure 2 $\mathrm{G}$ to J. To show how the coil pull back works, two iterations are presented in figure $2 \mathrm{G}$, corresponding to the $7^{\text {th }}$ (transparent coil inside the tube) and the $15^{t} h$ (outside the tube) iteration. When the coil is inside the tube, the algorithm pushes the tip towards the $\hat{z}$ axis. However, once the tip reaches the end of the tube, the algorithm progressively pulls back the tip until it is outside the tube, then the algorithm blocks the tube entrance by adding a potential field $\phi_{P B}=1$. Therefore, the algorithm selects a candidate location outside the tube, forcing the tip to follow another direction as it is presented with the iteration 15 . Figure $2 \mathrm{H}$, I and J present how the algorithm fully inserts the first, second and third coil, respectively. The final packing density inside the hexahedron was $23.7 \%$.

\section{Evaluation in Real Geometries}

To evaluate the results of our virtual coiling technique on patient-specific aneurysm geometries, two coiled aneurysms were selected. The first case was located at the right middle cerebral artery bifurcation and the second case at the anterior communicating artery. For each of these cases, a three dimensional 


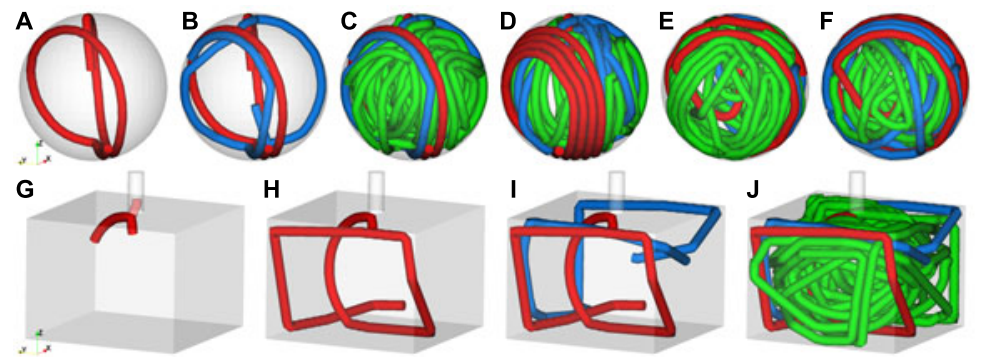

Fig. 2. Virtual coiling on idealized geometries. Test I is presented in (A) after one coil, (B) after two coils and (C) after three coils. (D), (E) and (F) show Test II, III and IV, respectively. Test $\mathrm{V}$ is depicted in $(\mathrm{G})$ for two iterations of the first coil, $(\mathrm{H})$ after one coil, (I) after two coils and $(\mathrm{J})$ after three coils.



Fig. 3. Comparison of a DSA and a virtual models of case 1, (A) and (B); and case 2, (C) and (D)

rotational angiography (3DRA) image before coiling and a digital subtraction angiography (DSA) image after coiling were acquired. Besides, the treatment information (number of coils and type) was recorded. For case 1, 11 coils were inserted with diameters between $0.245 \mathrm{~mm}$ and $0.29 \mathrm{~mm}$ and a total length of $67 \mathrm{~cm}$. In case 2,6 coils were inserted with diameter between $0.245 \mathrm{~mm}$ and $0.282 \mathrm{~mm}$ and a total length of $24 \mathrm{~cm}$.

Using the two selected cases, the following procedure was applied in each of them. The 3DRA image was segmented using a geodesic active region to get a 3D geometrical representation of the arteries and aneurysm [5]. Subsequently, the aneurysm geometry was extracted and isolated from the rest of the vasculature by placing a surface at its ostium. The virtual coiling technique was applied on the aneurysm model following the treatment procedure chosen for that case.

To compare the outcomes of the proposed technique in these realistic anatomies, a DSA image versus a virtual coiling image of the same case is presented in figure 3 A similar orientation of the 3D models was created, to match the virtual models with the DSA view. This orientation was created by a manual registration of the 3D geometries on the DSA image. In both cases, a similar filled area with coils was observed, although some wires produced particular differences.

Additional Cases: Figure 4 presents 10 additional patient-specific aneurysm models that were virtually treated. To apply our technique, each aneurysm was 

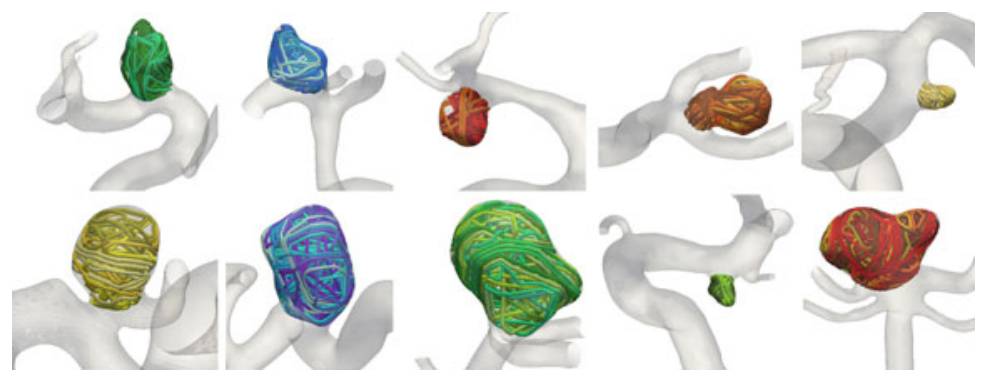

Fig. 4. Additional patient-specific aneurysm models

previously closed by adding a surface at its ostium. As it is visualized, the aneurysm location, morphology and size were different among cases. Besides, neither a DSA image sequence nor treatment data was available for these cases for comparison, and thereby, the presented results are meant for testing and visualization. Moreover, These results show the reliability of the technique when patient-specific models are used. The obtained packing densities were between $19 \%$ to $34.5 \%$.

\section{Discussion}

We have presented a technique to virtually coil intracranial aneurysms, based on a dynamic path planning algorithm to create the devices. This method relies on the facts that the final coil distribution is quasi-random, that currently it is not possible to reproduce exactly the same position and orientation of a real inserted coil inside a patient-specific aneurysm, and that clinicians aim at high packing densities when treating with coils [1].

The goal of this method is not for training, where physics-based models are essential [4]. Instead, we aim to understand the macroscopic behavior of the coil mesh, but considering the geometrical features of each coil. The simplicity of our technique allows reproducing realistic inserted coils and distribution and especially, obtaining similar results compared to physics-based techniques. Moreover, it produces high packing densities in patient-specific (34.5\%) and idealized models $(27.3 \%)$, which has been reported as a key parameter for coiling outcome [1].

The proposed method is fast to compute as it was observed from our results. The number of candidate locations is the most important parameter that impact the computational time. Elevating the number of candidate locations increases the computational time but also enhances the smoothness of the coils. Additionally, the computational time is proportional to the coil length, which clinically depends on the aneurysm size.

The application of our technique is focused on pre- and post-operative planning and diagnosis. For planning, the method calculates the packing density per 
coil, allows visualizing the coil distribution inside the aneurysm (angiographylike and 3D) and assessing the coiling outcome from a hemodynamics point of view using computational fluid dynamics solvers. For post-operative diagnosis, it is possible to assess for instance, the influence of different coil diameters or coil configurations on hemodynamics. Besides, the use of fluid-structure interaction methods could help to understand coil compaction phenomenon, which is one of the main causes of aneurysm recanalization after coiling.

\section{Conclusion}

We have described a technique for the virtual coiling of intracranial aneurysm, which can be used in either idealized geometries or patient-specific anatomies. The technique is based on a dynamic path planning algorithm to insert the coils. A comparison with DSA images for two real coiled aneurysm was performed and similar results were obtained. Additionally, 10 patient-specific aneurysm models were used show the reliability of the method in anatomical models.

Acknowledgments. The research leading to these results has received funding from the European Union Seventh Framework Programme (FP7/2007-2013) under grant agreement nr 223920, VPH-NoE project and has been partially funded by the Industrial and Technological Development Center (CDTI) under the CENIT-CDTEAM and CENIT-cvREMOD programs and by the European Commissions project @neurIST (IST-2005-027703). Alejandro F. F. is partially funded by the ICREA-Academia programme.

\section{References}

1. Li, M.H., Gao, B.L., Fang, C., Gu, B.X., Cheng, Y.S., Wang, W., Scotti, G.: Angiographic follow-up of cerebral aneurysms treated with Guglielmi detachable coils: an analysis of 162 cases with 173 aneurysms. AJNR Am. J. Neuroradiol. 27(5), 1107-1112 (2006)

2. Wootton, D.M., Ku, D.N.: Fluid Mechanics of Vascular Systems, Diseases, and Thrombosis. Annu. Rev. Biomed. Eng. 1, 299-329 (2003)

3. Larrabide, I., Kim, M., Augsburger, L., Villa-Uriol, M.C., Rufenacht, D., Frangi, A.F.: Fast virtual deployment of self-expandable stents: Method and in vitro evaluation for intracranial aneurysmal stenting. Med. Image Anal. (2010) (in press)

4. Dequidt, J., Marchal, M., Duriez, C., Kerien, E., Cotin, S.: Interactive simulation of embolization coils: Modeling and experimental validation. In: Metaxas, D., Axel, L., Fichtinger, G., Székely, G. (eds.) MICCAI 2008, Part I. LNCS, vol. 5241, pp. 695-702. Springer, Heidelberg (2008)

5. Bogunovic, H., Pozo, J.M., Villa-Uriol, M.C., Majoie, C.B., van den Berg, R., van Andel, H.A., Macho, J.M., Blasco, J., Roman, L.S., Frangi, A.F.: Automated segmentation of cerebral vasculature with aneurysms in 3DRA and TOF-MRA using geodesic active regions: an evaluation study. Med. Phys. 38(1), 210-222 (2011) 